\title{
Modified Projectile Linear Theory for Rapid Trajectory Prediction
}

\author{
Leonard C. Hainz III* and Mark Costello ${ }^{\dagger}$ \\ Oregon State University, Corvallis, Oregon 97331
}

\begin{abstract}
In some smart weapons, estimation of the impact point of the shell at each computation cycle of the control law is an integral part of the control strategy. In these situations, the impact point predictor is part of the imbedded computing system onboard the projectile. Practical considerations dictate that the impact point predictor yield rapid yet reasonably accurate estimates. Common methods for rapid trajectory prediction are numerical integration of point mass dynamic equations and evaluation of approximate closed-form solutions of the rigid-body projectile dynamic equations. These methods are shown to exhibit poor impact point prediction for long-range shots with high gun elevations characteristic of smart indirect fire munitions. Through modifications of projectile linear theory, a rapid projectile impact point predictor is proposed that eliminates the accuracy problems of the other methods while preserving low computational requirements. Typical results are provided for a short-range trajectory of a direct fire fin-stabilized projectile and a long-range trajectory for an indirect fire spin-stabilized round to substantiate these claims.
\end{abstract}

\section{Nomenclature}

$A, B, C, E$

$a_{V}, b_{V}$

$C_{D D}$

$C_{L P}$

$C_{M Q}$

$C_{N A}$

$C_{N P A}$

$C_{N R}$

$C_{X 0}$

$C_{X 2}$

$C_{Y B 1}$

$C_{Y 0}$

$C_{Z A 1}$

$C_{Z 0}$

$D$

$F_{v}, F_{w}, F_{q}, F_{r}$

$g$

I

$I_{X X}, I_{Y Y}, I_{Z Z}$

$I_{X Y}, I_{Y Z}, I_{X Z}$

$\tilde{L}, \tilde{M}, \tilde{N}$

$\tilde{L}_{A}, \tilde{M}_{A}, \tilde{N}_{A}$

$=$ epicyclic matrix coefficients

$=$ velocity solution coefficients

$=$ roll moment aerodynamic coefficient due to fin cant

$=$ roll damping aerodynamic coefficient

$=$ pitch damping aerodynamic coefficient

$=$ normal force aerodynamic coefficient

$=$ Magnus force aerodynamic coefficient

= yaw damping aerodynamic coefficient

= zero yaw axial force aerodynamic coefficient

$=$ yaw angle squared axial force aerodynamic coefficient

$=$ normal force aerodynamic coefficient along $j_{n}$ axis

$=$ trim side force aerodynamic coefficient

$=$ normal force aerodynamic coefficient along $k_{n r}$ axis

$=$ trim vertical force aerodynamic coefficient

$=$ projectile reference diameter

$=$ epicyclic equations forcing coefficients

$=$ gravity

$=$ projectile inertia matrix

$=$ diagonal components of the inertia matrix

$=$ off-diagonal components of the inertia matrix

$=$ external moments on projectile, expressed in the no-roll frame

$=$ moment due to aerodynamic force, expressed in the no-roll frame

$\tilde{L}_{M}, \tilde{M}_{M}, \tilde{N}_{M} \quad=$ moment due to Magnus force, expressed in the no-roll frame

$\tilde{L}_{\mathrm{UA}}, \tilde{M}_{\mathrm{UA}}, \tilde{N}_{\mathrm{UA}}=$ unsteady aerodynamic moment, expressed in the no-roll frame

Received 11 March 2004; revision received 15 February 2005; accepted for publication 22 February 2005. Copyright (C) 2005 by the American Institute of Aeronautics and Astronautics, Inc. All rights reserved. Copies of this paper may be made for personal or internal use, on condition that the copier pay the $\$ 10.00$ per-copy fee to the Copyright Clearance Center, Inc., 222 Rosewood Drive, Danvers, MA 01923; include the code 0731-5090/05 $\$ 10.00$ in correspondence with the CCC.

*Research Assistant, Department of Mechanical Engineering; currently Engineer, Missile Flight Control, Orbital Sciences Corporation, Chandler, AZ 85248.

${ }^{\dagger}$ Associate Professor, Department of Mechanical Engineering. Member AIAA. $\tilde{p}$

$\tilde{q}, \tilde{r}$

$\boldsymbol{R}_{\oplus C A}$

$\boldsymbol{R}_{\oplus C M}$

$R_{M C M}$

$R_{M C P}$

$s$

$t$

$\tilde{u}, \tilde{v}, \tilde{w}$

$\tilde{u}_{A}, \tilde{v}_{A}, \tilde{w}_{A}$

$\tilde{u}_{W}, \tilde{v}_{W}, \tilde{w}_{W}$

$V$

$V_{\tilde{X}}$

$\tilde{X}, \tilde{Y}, \tilde{Z}$

$\tilde{X}_{A}, \tilde{Y}_{A}, \tilde{Z}_{A}$

$\tilde{X}_{M}, \tilde{Y}_{M}, \tilde{Z}_{M}$

$\tilde{X}_{w}, \tilde{Y}_{w}, \tilde{Z}_{w}$

$x, y, z$

$\alpha, \beta$

$\theta, \psi$

$\rho$

$\sigma_{F}, \sigma_{S}$

$\Phi_{F}, \Phi_{S}$

$\phi$

\section{Introduction}

B EFORE 1500, it was believed that projectiles in flight moved in a straight line until the round lost its "impetus" at which point it fell to the ground abruptly. In the first half of the 16th century, Italian ballistician Niccolo Tartaglia first noted that projectiles in flight move along a curved path. The work of Galileo Galilei, the well-known Italian physicist and astronomer, generated the first 
analytic projectile trajectory solution and found that the path was parabolic. He effectively modeled the projectile as a point mass with only gravity acting on the projectile. His trajectory predictions were remarkably accurate primarily because early projectiles were heavy and fired with low initial velocity. In 1711, Johann Bernoulli created the first projectile trajectory solution including the effect of aerodynamic drag. Understanding of the effect of aerodynamic drag on the trajectory of a projectile was greatly aided by the invention of the ballistic pendulum by English ballistician Benjamin Robins in early part of the 18th century. The ballistic pendulum allowed the impact velocity of a projectile to be determined. When rounds were fired at the pendulum from different distances, the effect of drag was deduced. A variety of functions relating drag and projectile velocity were developed by different research organizations. In the middle of the 18th century, Swiss mathematician Leonard Euler developed the short arc method for rapidly obtaining approximate solutions of the point mass projectile dynamic equations with aerodynamic drag as a function of velocity. This basic method of generating projectile trajectories remained the state of the art until World War I. During this time, various organizations created general tables providing impact data for a given shell under different muzzle velocity and angle of launch conditions. One of the more notable set of tables was generated by Italian ballistician Francesco Siacci. As new gun technology emerged in the form of rifled guns (1750) and elongated bullets (1825) and new missions such as artillery and antiaircraft surfaced, problems with the point mass projectile dynamic model mounted. The basic point mass model was not able to account for swerve due to the yaw of repose or drag and lift caused by yawing of elongated projectiles. Moreover, the point mass model did not provide a basis for stability of a round in flight. To eliminate some of the problems associated with point mass projectile dynamic modeling, the modified point mass model was developed, which included yaw of repose in an approximate manner. ${ }^{1}$

More than 80 years ago, pioneering English ballisticians Fowler, Gallop, Lock, and Richmond constructed the first rigid six-degreeof-freedom projectile exterior ballistics model. ${ }^{2}$ Their model contained a reasonably complete aerodynamic force and moment expansion for a spinning shell and included aerodynamic damping along with Magnus force and moment. Guided by an extensive set of yaw card firings, these researchers also created the first approximate analytic solution of the six-degree-of-freedom projectile equations of motion by introducing a set of simplifications based on the relative size of different dynamic quantities of a stable projectile and based on clever linearization by artificially separating the dynamic equations into uncoupled groups. The resulting theory is commonly called projectile linear theory. Refinements and improvements to projectile linear theory were made by Kent, ${ }^{3}$ Neilson and Synge, ${ }^{4}$ Kelley and McShane, ${ }^{5}$ and Kelley et al. ${ }^{6}$ Projectile linear theory has proved an invaluable tool in understanding basic dynamic characteristics of projectiles in atmospheric flight, for establishing stability criteria for fin- and spin-stabilized projectiles, and for extracting projectile aerodynamic loads from spark range data. Various authors have extended projectile linear theory to accommodate asymmetric mass properties, ${ }^{7}$ fluid payloads, ${ }^{8,9}$ moving internal parts, ${ }^{10-12}$ ascending flight,${ }^{13}$ lateral force impulses,${ }^{14,15}$ and dual-spin projectiles. ${ }^{16,17}$

Whereas point mass and approximate rigid-projectile solutions provide qualitatively correct trajectories, impact point prediction errors can be relatively large, particularly for high launch angles and long-range trajectories. For the design of some smart weapon flight control systems, prediction of the impact point during flight is an integral part of a complex control law. ${ }^{18}$ When an array of different sensors is used, an observer is used first to obtain an estimate of the projectile state at a given time instant. Miss distance is then estimated by projecting the bullet from an arbitrary state to impact using a dynamic model solution. Common methods for predicting impact point include numerical integration of three-degree-of-freedom point mass models, numerical integration of four-degree-of-freedom modified point mass models, and approximate analytical solutions of six-degree-of-freedom rigid-projectile models. The work reported here documents several modifications to standard projectile linear theory that significantly improve accuracy of impact point prediction while still maintaining low computation overhead required for real-time implementation of a smart weapon control law. Results using the modified projectile linear theory trajectory generator are contrasted against other common trajectory generation techniques.

\section{Projectile Dynamic Model}

A six-degree-of-freedom rigid-projectile model is employed to predict the dynamics of a projectile in flight. These equations assume a flat Earth. The six degrees of freedom comprise the three translational components describing the position of the projectile's center of mass, and the three Euler angles describing the orientation of the projectile with respect to Figs. 1 and 2 provide a visualization of the degrees of freedom. The equations of motion for the six-degree-of-freedom model, derived in the no-roll frame, are

$$
\begin{gathered}
\left\{\begin{array}{c}
\dot{x} \\
\dot{y} \\
\dot{z}
\end{array}\right\}=\left[\begin{array}{ccc}
c_{\theta} c_{\psi} & -s_{\psi} & s_{\theta} c_{\psi} \\
c_{\theta} s_{\psi} & c_{\psi} & s_{\theta} s_{\psi} \\
-s_{\theta} & 0 & c_{\theta}
\end{array}\right]\left\{\begin{array}{c}
\tilde{u} \\
\tilde{v} \\
\tilde{w}
\end{array}\right\} \\
\left\{\begin{array}{c}
\dot{\phi} \\
\dot{\theta} \\
\dot{\psi}
\end{array}\right\}=\left[\begin{array}{ccc}
1 & 0 & t_{\theta} \\
0 & 1 & 0 \\
0 & 0 & 1 / c_{\theta}
\end{array}\right]\left\{\begin{array}{l}
\tilde{p} \\
\tilde{q} \\
\tilde{r}
\end{array}\right\}
\end{gathered}
$$

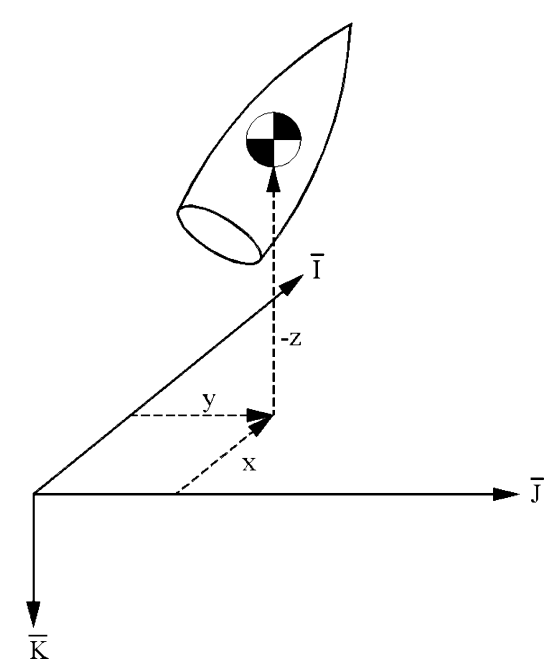

Fig. 1 Projectile position coordinate definitions.

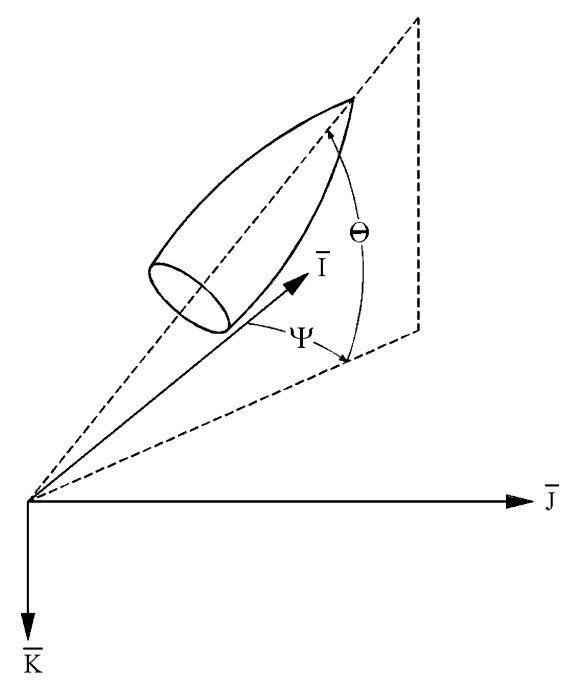

Fig. 2 Projectile orientation definitions. 


$$
\begin{gathered}
\left\{\begin{array}{c}
\dot{\tilde{u}} \\
\dot{\tilde{v}} \\
\dot{\tilde{w}}
\end{array}\right\}=\left\{\begin{array}{c}
\tilde{X} / m \\
\tilde{Y} / m \\
\tilde{Z} / m
\end{array}\right\}+\left\{\begin{array}{c}
\tilde{r} \tilde{v}-\tilde{q} \tilde{w} \\
-t_{\theta} \tilde{r} \tilde{w}-\tilde{r} \tilde{u} \\
\tilde{q} \tilde{u}+t_{\theta} \tilde{r} \tilde{v}
\end{array}\right\} \\
\left\{\begin{array}{c}
\dot{\tilde{p}} \\
\dot{\tilde{q}} \\
\dot{r}
\end{array}\right\}=[I]^{-1}\left(\left\{\begin{array}{c}
\tilde{L} \\
\tilde{M} \\
\tilde{N}
\end{array}\right\}-\left[\begin{array}{ccc}
0 & -\tilde{r} & \tilde{q} \\
\tilde{r} & 0 & \tilde{r} t_{\theta} \\
-\tilde{q} & -\tilde{r} t_{\theta} & 0
\end{array}\right][I]\left\{\begin{array}{c}
\tilde{p} \\
\tilde{q} \\
\tilde{r}
\end{array}\right\}\right)
\end{gathered}
$$

The force acting on the projectile in Eq. (3) comprises the weight force $W$ and the aerodynamic force. The aerodynamic force is split into a standard $A$ and Magnus $M$ aerodynamic force. The combination of forces is

$$
\left\{\begin{array}{c}
\tilde{X} \\
\tilde{Y} \\
\tilde{Z}
\end{array}\right\}=\left\{\begin{array}{c}
\tilde{X}_{w} \\
\tilde{Y}_{w} \\
\tilde{Z}_{w}
\end{array}\right\}+\left\{\begin{array}{c}
\tilde{X}_{A} \\
\tilde{Y}_{A} \\
\tilde{Z}_{A}
\end{array}\right\}+\left\{\begin{array}{c}
\tilde{X}_{M} \\
\tilde{Y}_{M} \\
\tilde{Z}_{M}
\end{array}\right\}
$$

The weight force in the no-roll coordinate system is expressed as

$$
\left\{\begin{array}{c}
\tilde{X}_{w} \\
\tilde{Y}_{w} \\
\tilde{Z}_{w}
\end{array}\right\}=m g\left\{\begin{array}{c}
-s_{\theta} \\
0 \\
c_{\theta}
\end{array}\right\}
$$

The aerodynamic force in the no-roll coordinate system, which acts on the projectile at the aerodynamic center of pressure, is

$$
\left\{\begin{array}{c}
\tilde{X}_{A} \\
\tilde{Y}_{A} \\
\tilde{Z}_{A}
\end{array}\right\}=-\frac{\pi}{8} \rho V_{A}^{2} D^{2}\left\{\begin{array}{c}
C_{X 0}+C_{X 2} \alpha^{2}+C_{X 2} \beta^{2} \\
C_{Y 0}+C_{Y B 1} \beta \\
C_{Z 0}+C_{Z A 1} \alpha
\end{array}\right\}
$$

The Magnus force in the no-roll coordinate system, which acts on the projectile at the Magnus force center of pressure is

$$
\left\{\begin{array}{c}
\tilde{X}_{M} \\
\tilde{Y}_{M} \\
\tilde{Z}_{M}
\end{array}\right\}=\frac{\pi}{8} \rho V_{A}^{2} D^{2}\left\{\begin{array}{c}
0 \\
\frac{\tilde{p} D C_{N P A} \alpha}{2 V_{A}} \\
\frac{-\tilde{p} D C_{N P A} \beta}{2 V_{A}}
\end{array}\right\}
$$

Equations (7) and (8) are based on Mach number-dependent coefficients, the aerodynamic angles of attack given in Eqs. (9) and (10), and the total aerodynamic velocity given in Eq. (11) as follows:

$$
\begin{array}{r}
\alpha=\tan ^{-1}\left(\tilde{w}_{A} / \tilde{u}_{A}\right) \\
\beta=\tan ^{-1}\left(\tilde{v}_{A} / \tilde{u}_{A}\right) \\
V_{A}=\sqrt{\tilde{u}_{A}^{2}+\tilde{v}_{A}^{2}+\tilde{w}_{A}^{2}}
\end{array}
$$

The moment acting on the projectile in Eq. (4) comprises the moment due to the standard aerodynamic force $A$, the moment due to the Magnus aerodynamic force $M$, and the unsteady aerodynamic moment (UA),

$$
\left\{\begin{array}{c}
\tilde{L} \\
\tilde{M} \\
\tilde{N}
\end{array}\right\}=\left\{\begin{array}{c}
\tilde{L}_{A} \\
\tilde{M}_{A} \\
\tilde{N}_{A}
\end{array}\right\}+\left\{\begin{array}{c}
\tilde{L}_{M} \\
\tilde{M}_{M} \\
\tilde{N}_{M}
\end{array}\right\}+\left\{\begin{array}{c}
\tilde{L}_{\mathrm{UA}} \\
\tilde{M}_{\mathrm{UA}} \\
\tilde{N}_{\mathrm{UA}}
\end{array}\right\}
$$

The moment due to the aerodynamic force is

$$
\left\{\begin{array}{l}
\tilde{L}_{A} \\
\tilde{M}_{A} \\
\tilde{N}_{A}
\end{array}\right\}=\left[\begin{array}{ccc}
0 & -R_{\oplus C A Z} & R_{\oplus C A Y} \\
R_{\oplus C A Z} & 0 & -R_{\oplus C A X} \\
-R_{\oplus C A Y} & R_{\oplus C A X} & 0
\end{array}\right]\left\{\begin{array}{l}
\tilde{X}_{A} \\
\tilde{Y}_{A} \\
\tilde{Z}_{A}
\end{array}\right\}
$$

The moment due to the Magnus force is

$$
\left\{\begin{array}{c}
\tilde{L}_{M} \\
\tilde{M}_{M} \\
\tilde{N}_{M}
\end{array}\right\}=\left[\begin{array}{ccc}
0 & -R_{\oplus C M Z} & R_{\oplus C M Y} \\
R_{\oplus C M Z} & 0 & -R_{\oplus C M X} \\
-R_{\oplus C M Y} & R_{\oplus C M X} & 0
\end{array}\right]\left\{\begin{array}{l}
\tilde{X}_{M} \\
\tilde{Y}_{M} \\
\tilde{Z}_{M}
\end{array}\right\}
$$

The unsteady aerodynamic moments acting on the projectile are

$$
\left\{\begin{array}{c}
\tilde{L}_{\mathrm{UA}} \\
\tilde{M}_{\mathrm{UA}} \\
\tilde{N}_{\mathrm{UA}}
\end{array}\right\}=\frac{\pi}{8} \rho V_{A}^{2} D^{3}\left\{\begin{array}{c}
C_{D D}+\frac{\tilde{p} D C_{L P}}{2 V_{A}} \\
\frac{\tilde{q} D C_{M Q}}{2 V_{A}} \\
\frac{\tilde{r} D C_{N R}}{2 V_{A}}
\end{array}\right\}
$$

The coefficients used in this model are projectile-specific functions of the Mach number of the projectile. For fin-stabilized projectiles, Magnus force and moment are typically ignored because their effect is rather small for slowing rolling projectiles.

The dynamic equations of motion (1-15) are highly nonlinear. Thus, numerical integration is commonly used to obtain solutions to this initial value problem.

\section{Modified Projectile Linear Theory}

Mathematically, the projectile dynamic model just discussed consists of 12 highly nonlinear ordinary differential equations. These equations are not amenable to a closed-form solution. Simplifications to the dynamic equations have been identified over time that yield an analytically solvable set of quasi-linear differential equations that enable rapid trajectory construction. Projectile linear theory provides reasonably accurate trajectory prediction for flat fire short trajectories. For high quadrant elevation shots or long-distance trajectories, qualitatively correct trajectories are generated. However, in these cases, significant impact point prediction errors are noticed with conventional projectile linear theory. To improve trajectory prediction, the solution procedure is altered, and the typical assumption of small Euler pitch angle is relaxed. To develop the modified projectile linear theory equations, the following set of simplifications are employed:

1) The station line velocity $\tilde{u}$, roll rate $\tilde{p}$, and roll angle $\phi$ are large in relation to the side velocities $\tilde{v}$ and $\tilde{w}$, yaw angle $\psi$, pitch and yaw rates $\tilde{q}$ and $\tilde{r}$, and wind velocity components $\tilde{u}_{W}, \tilde{v}_{W}$, and $\tilde{w}_{W}$. Products of small values and derivatives of small values are treated as negligible.

2) The yaw angle $\psi$ is small, allowing the simplifications

$$
\sin (\psi) \approx \psi, \quad \cos (\psi) \approx 1
$$

to hold

3) The aerodynamic angles of attack are also small in magnitude, allowing the angles of attack to be as

$$
\begin{gathered}
\alpha \approx \tilde{w} / V \\
\beta \approx \tilde{v} / V
\end{gathered}
$$

instead of Eqs. (9) and (10).

4) The Magnus force components are small in comparison with the weight and aerodynamic force components, and so they are treated as negligible in the modified linear theory force computations. The Magnus force does create a nonnegligible moment, and so it is maintained in the moment computations.

5) The projectile is geometrically symmetrical about the station line. This allows the inertia matrix to be simplified as

$$
\begin{array}{r}
I_{X Y}=I_{Y Z}=I_{X Z}=0 \\
I_{Y Y}=I_{Z Z}
\end{array}
$$


6) The projectile is aerodynamically symmetric. This allows the aerodynamic coefficients to be simplified as

$$
\begin{gathered}
C_{M Q}=C_{N R} \\
C_{Y 0}=C_{Z 0}=0 \\
C_{Y B 1}=C_{Z B 1}=C_{N A}
\end{gathered}
$$

This also allows for a simplification in the expressions of the distances from the center of mass to both the standard aerodynamic and Magnus centers of pressure as

$$
\begin{array}{cl}
R_{\oplus C A X}=R_{M C P}, & R_{\oplus C M X}=R_{M C M} \\
R_{\oplus C A Y}=R_{\oplus C A Z}=0, & R_{\oplus C M Y}=R_{\oplus C M Z}=0
\end{array}
$$

7) The wind velocity component $\tilde{u}_{W}$ parallel to the projectile station line is negligible in comparison to the projectile total velocity.

8) A change of variables is introduced in which the station line velocity $\tilde{u}$ is replaced by the projectile total velocity $V$ the projectile total velocity and the application of assumption 1 is expressed as

$$
V=\sqrt{\tilde{u}^{2}+\tilde{v}^{2}+\tilde{w}^{2}} \approx \tilde{u}
$$

The time derivative of the total velocity and the application of assumption 1 is expressed as

$$
\dot{V}=(\tilde{u} \dot{\tilde{u}}+\tilde{v} \dot{\tilde{v}}+\tilde{w} \dot{\tilde{w}}) / V \approx \dot{\tilde{u}}
$$

9) Another change of variables is performed to convert the independent variable from time $t$ to dimensionless arc length $s$. The arc length is dimensionless and expresses the projectiles downrange travel in calibers:

$$
s=\frac{1}{D} \int_{0}^{t} V \mathrm{~d} \tau
$$

The following equations express the relationships between time derivatives and arc-length derivatives in terms of an example variable $\zeta$, where prime terms are used to denote arc-length derivatives and dotted terms denote time derivatives:

$$
\begin{gathered}
\dot{\zeta}=(V / D) \zeta^{\prime} \\
\ddot{\zeta}=(V / D)^{2}\left(\zeta^{\prime \prime}+\zeta^{\prime} V^{\prime} / V\right)
\end{gathered}
$$

Applying the previous assumptions and transformations to the no-roll frame six-degree-of-freedom equations yields

$x^{\prime}=c_{\theta} D$

$y^{\prime}=c_{\theta} D \psi+\frac{D}{V} \tilde{v}$

$z^{\prime}=-D s_{\theta}+\frac{D c_{\theta}}{V} \tilde{w}$

$\phi^{\prime}=\frac{D}{V} \tilde{p}$

$\theta^{\prime}=\frac{D}{V} \tilde{q}$

$\psi^{\prime}=\frac{D}{V c_{\theta}} \tilde{r}$

$V^{\prime}=-\frac{\pi \rho D^{3}}{8 m} C_{X 0} V-\frac{D g}{V} s_{\theta}$

$\tilde{v}^{\prime}=-\frac{\pi \rho D^{3}}{8 m} C_{N A}\left(\tilde{v}-\tilde{v}_{W}\right)-D \tilde{r}$

$\tilde{w}^{\prime}=-\frac{\pi \rho D^{3}}{8 m} C_{N A}\left(\tilde{w}-\tilde{w}_{W}\right)+D \tilde{q}+\frac{D g c_{\theta}}{V}$

$\tilde{p}^{\prime}=\frac{\pi \rho V D^{4}}{8 I_{X X}} C_{D D}+\frac{\pi \rho D^{5}}{16 I_{X X}} C_{L P} \tilde{p}$

$$
\begin{aligned}
\tilde{q}^{\prime}= & \frac{\pi \rho D^{4} R_{M C M}}{16 I_{Y Y} V} C_{Y P A} \tilde{p}\left(\tilde{v}-\tilde{v}_{W}\right)+\frac{\pi \rho D^{3} R_{M C P}}{8 I_{Y Y}} C_{N A}\left(\tilde{w}-\tilde{w}_{W}\right) \\
& +\frac{\pi \rho D^{5}}{16 I_{Y Y}} C_{M Q} \tilde{q}-\frac{I_{X X} D}{I_{Y Y} V} \tilde{p} \tilde{r}_{q} \\
\tilde{r}^{\prime}= & -\frac{\pi \rho D^{3} R_{M C P}}{8 I_{Y Y}} C_{N A}\left(\tilde{v}-\tilde{v}_{W}\right)+\frac{\pi \rho D^{4} R_{M C M}}{16 I_{Y Y} V} C_{Y P A} \tilde{p}\left(\tilde{w}-\tilde{w}_{W}\right) \\
& +\frac{I_{X X} D}{I_{Y Y} V} \tilde{p} \tilde{q}+\frac{\pi \rho D^{5}}{16 I_{Y Y}} C_{M Q} \tilde{r}
\end{aligned}
$$

Equations (30-41) form the modified linear theory equations of motion for a projectile. These equations, although not strictly linear, are in a form that makes an approximate analytical closed-form solution possible. In standard projectile linear theory, the additional assumption of small Euler pitch angle is made, leading to $\sin \theta \approx \theta$ and $\cos \theta \approx 1$.

\section{Analytical Closed-Form Solution}

The closed-form solutions of Eqs. (30-41) are aided by making the following assumptions:

1) The aerodynamic coefficients are constant.

2) The total velocity $V$ is slowly changing with relation to the other variables. It is only treated as a dynamic variable in the solution of the total velocity equation (36).

3 ) The roll rate $\tilde{p}$ is slowly changing in relation to the other angular rates. It is treated as a dynamic variable in the solution of the roll rate equation, but as a constant in the solution of the epicyclic equations. With these assumptions, the equations of motion decouple into five groups of equations that are largely uncoupled, namely, the total velocity, roll rate, epicyclic, Euler angle, and swerve equations. The solution process assumes that the epicyclic variables change most rapidly, followed by the total velocity and roll rate.

\section{Total Velocity Solution}

The total velocity solution is found by treating the pitch angle as a constant and then integrating Eq. (36):

$$
V(s)=\sqrt{\left(V_{0}^{2}+b_{V} / a_{V}\right) e^{-2 a_{V} s}-b_{V} / a_{V}}
$$

The coefficients appearing in Eq. (42) are

$$
a_{V}=\left(\pi \rho D^{3} / 8 m\right) C_{X 0}, \quad b_{V}=g D \sin \left(\theta_{0}\right)
$$

\section{Roll Rate Solution}

The roll rate solution is

$$
\tilde{p}(s)=C_{p e 1} e^{C_{p e 2} s}-C_{p 0}
$$

where

$$
C_{p 0}=\frac{2 C_{D D} V_{0}}{D C_{L P}}, \quad C_{p e 1}=\tilde{p}_{0}+\frac{2 C_{D D} V_{0}}{D C_{L P}}, \quad C_{p e 2}=\frac{\pi \rho D^{5}}{16 I_{X X}} C_{L P}
$$

\section{Epicyclic Solution}

The remaining velocity and angular rate equations (37) and (38) and (40) and (41) form a set of coupled nonhomogeneous differential equations referred to as the epicyclic equations. If the pitch angle, total velocity, and roll rate are treated as constant, these equations are linear and can be compacted into the form

$$
\left\{\begin{array}{c}
\tilde{v}^{\prime} \\
\tilde{w}^{\prime} \\
\tilde{q}^{\prime} \\
\tilde{r}^{\prime}
\end{array}\right\}=\left[\begin{array}{cccc}
-A & 0 & 0 & -D \\
0 & -A & D & 0 \\
B / D & C / D & E & -F \\
-C / D & B / D & F & E
\end{array}\right]\left\{\begin{array}{c}
\tilde{v} \\
\tilde{w} \\
\tilde{q} \\
\tilde{r}
\end{array}\right\}+\left\{\begin{array}{l}
F_{v} \\
F_{w} \\
F_{q} \\
F_{r}
\end{array}\right\}
$$

The coefficients appearing in Eq. (46) are expressed in

$$
A=\frac{\pi \rho D^{3}}{8 m} C_{N A}
$$




$$
\begin{gathered}
B=\frac{\pi \rho D^{5} R_{M C M}}{16 I_{Y Y} V_{0}} C_{Y P A} \tilde{p} \\
C=\frac{\pi \rho D^{4} R_{M C P}}{8 I_{Y Y}} C_{N A} \\
E=\frac{\pi \rho D^{5}}{16 I_{Y Y}} C_{M Q} \\
F=\frac{I_{X X} D}{I_{Y Y} V_{0}} \tilde{p} \\
F_{v}=A \tilde{v}_{\text {wind }} \\
F_{w}=A \tilde{w}_{\text {wind }}+\frac{D g c_{\theta_{0}}}{V_{0}} \\
F_{q}=-\frac{B}{D} \tilde{v}_{\text {wind }}-\frac{C}{D} \tilde{w}_{\text {wind }} \\
F_{r}=-\frac{B}{D} \tilde{w}_{\text {wind }}+\frac{C}{D} \tilde{v}_{\text {wind }}
\end{gathered}
$$

The eigenvalues for this system split into two complex pairs, commonly called the fast and slow modes. The coupled set of differential equations is solved using matrix Laplace transformation. The closed-form solutions for the side velocities, pitch rate, and yaw rate are

$$
\begin{aligned}
\tilde{v}(s) & =C_{v 0}+e^{\sigma_{F} s}\left[C_{v f c} \cos \left(\Phi_{F} s\right)+C_{v f s} \sin \left(\Phi_{F} s\right)\right] \\
& +e^{\sigma_{S} s}\left[C_{v s c} \cos \left(\Phi_{S} s\right)+C_{v s s} \sin \left(\Phi_{S} s\right)\right] \\
\tilde{w}(s) & =C_{w 0}+e^{\sigma_{F} s}\left[C_{w f c} \cos \left(\Phi_{F} s\right)+C_{w f s} \sin \left(\Phi_{F} s\right)\right] \\
& +e^{\sigma_{S} s}\left[C_{w s c} \cos \left(\Phi_{S} s\right)+C_{w s s} \sin \left(\Phi_{S} s\right)\right] \\
\tilde{q}(s) & =C_{q 0}+e^{\sigma_{F} s}\left[C_{q f c} \cos \left(\Phi_{F} s\right)+C_{q f s} \sin \left(\Phi_{F} s\right)\right] \\
& +e^{\sigma_{S} s}\left[C_{q s c} \cos \left(\Phi_{S} s\right)+C_{q s s} \sin \left(\Phi_{S} s\right)\right] \\
\tilde{r}(s) & =C_{r 0}+e^{\sigma_{F} s}\left[C_{r f c} \cos \left(\Phi_{F} s\right)+C_{r f s} \sin \left(\Phi_{F} s\right)\right] \\
& +e^{\sigma_{S} s}\left[C_{r s c} \cos \left(\Phi_{S} s\right)+C_{r s s} \sin \left(\Phi_{S} s\right)\right]
\end{aligned}
$$

The expressions for the coefficients expressed in Eqs. (56-65) are extensive and not included in this paper due to length restrictions. However, these coefficients are algebraic equations that are easily evaluated inside a computer code.

\section{Euler Angle and Swerve Solution}

The remaining states are computed through integration of the solutions expressed in Eqs. (42), (44), and (56-59). The variables appear in a linear fashion, after applying the earlier discussed assumptions, with the exception of pitch angle $\theta$, which is operated on by trigonometric functions and appears in terms with other independent variables. In terms in which a trigonometric function of the pitch angle appears as the only independent variable, the integral is approximated by the trapezoid method. When pitch angle appears in a term containing another independent variable, pitch angle is treated as a constant,

$$
\begin{aligned}
\phi(s) & =C_{\phi 0}+C_{\phi 1} s+C_{\phi e 1} e^{C_{\phi e 2} s} \\
\theta(s) & =C_{\theta 0}+C_{\theta 1} s+e^{\sigma_{F} s}\left[C_{\theta f c} \cos \left(\Phi_{F} s\right)+C_{\theta f s} \sin \left(\Phi_{F} s\right)\right] \\
& +e^{\sigma_{S} s}\left[C_{\theta s c} \cos \left(\Phi_{S} s\right)+C_{\theta s s} \sin \left(\Phi_{S} s\right)\right] \\
\psi(s) & =C_{\psi 0}+C_{\psi 1} s+e^{\sigma_{F} s}\left[C_{\psi f c} \cos \left(\Phi_{F} s\right)+C_{\psi f s} \sin \left(\Phi_{F} s\right)\right] \\
& +e^{\sigma_{S} s}\left[C_{\psi s c} \cos \left(\Phi_{S} s\right)+C_{\psi s s} \sin \left(\Phi_{S} s\right)\right] \\
x(s) & =x_{0}+\frac{1}{2} D s\left\{\cos [\theta(s)]+\cos \left(\theta_{0}\right)\right\} \\
y(s) & =C_{y 0}+C_{y 1} s+C_{y 2} s^{2}+e^{\sigma_{F} s}\left[C_{y f c} \cos \left(\Phi_{F} s\right)\right. \\
& \left.+C_{y f s} \sin \left(\Phi_{F} s\right)\right]+e^{\sigma_{S} s}\left(C_{y s c} \cos \left(\Phi_{S} s\right)+C_{y s s} \sin \left(\Phi_{S} s\right)\right]
\end{aligned}
$$

$$
\begin{aligned}
z(s) & =C_{z 0}+C_{z 1} s-\frac{1}{2} D s\left\{\sin [\theta(s)]+\sin \left(\theta_{0}\right)\right\} \\
& +e^{\sigma_{F} s}\left[C_{z f c} \cos \left(\Phi_{F} s\right)+C_{z f s} \sin \left(\Phi_{F} s\right)\right] \\
& +e^{\sigma_{s} s}\left[C_{z s c} \cos \left(\Phi_{S} s\right)+C_{z s s} \sin \left(\Phi_{S} s\right)\right]
\end{aligned}
$$

\section{Solution Implementation}

Because of the variation of coefficient values $\left(C_{i}, \Phi_{i}\right.$, etc. $)$ that are treated as constant in the closed-form solution, inaccuracies build in the solution, especially when considering long-range indirect fire trajectories. To minimize these inaccuracies, the model is periodically updated along the trajectory. In this process, the last trajectory point computed is treated as the initial conditions for a new trajectory segment. Before the new trajectory segment is computed, all constants in the model are recomputed using the new initial condition data.

Current models employed for rapid trajectory prediction include three-degree-of-freedom point mass models, four-degree-offreedom modified point mass models, and conventional projectile linear theory. These methods are limited in their ability to predict accurately a projectile trajectory under general conditions. Point mass models account for high launch angle trajectories, but ignore rotational dynamics, leading to poor swerve prediction. The standard linear theory solution includes rotational dynamics, but is limited to low launch angles. The modified linear theory closed-form solution expressed in Eqs. (42), (44), and (56-65) allows rapid and accurate calculation of long-range trajectories with large pitch angles.

\section{Results}

The capabilities of modified projectile linear theory trajectory generation are highlighted using simulation for two example scenarios. Although not intended to be exhaustive, these scenarios underline the merit of the reported approach. In each case the results of modified linear theory are compared to results generated by existing models to allow a baseline for comparison. The first case simulates the short-range trajectory of a fin-stabilized projectile launched at low pitch angle. The second case simulates a relatively long-range trajectory of a spin-stabilized projectile launched at high pitch angle. For each case, typical trajectories were computed using five methods: numerical integration of the nonlinear six-degree-of-freedom rigid-projectile equations, numerical integration of the four-degreeof-freedom modified point mass equations, numerical integration of the three-degree-of-freedom point mass equations, analytic solution of the six-degree-of-freedom approximate equations from standard projectile linear theory, and analytic solution of the six-degree-offreedom approximate equations from modified projectile linear theory. The nonlinear six-degree-of-freedom numerical simulation is used as the truth model for validating the other methods. This projectile dynamic model has been previously shown to agree favorably with spark range data. ${ }^{19}$ The three-degree-of-freedom point mass model treats the projectile as a point mass and applies the zero-angleof-attack axial aerodynamic force component directly opposing the aerodynamic velocity. The four-degree-of-freedom modified point mass model is an extension of the three-degree-of-freedom point mass model that adds the uncoupled roll equation to the model and also adds a forcing term in point mass equations to account for yaw of repose. The yaw of repose forcing term is extracted from the standard projectile linear theory swerve equations. The standard projectile linear theory solution is derived and computed similarly to the described modified projectile linear theory solution with differences in the determination of the closed-form solutions stemming from the assumption of small Euler pitch angle. Note that standard projectile linear theory does include yaw of repose. In Ref. 1, details are provided on all models discussed except for the modified projectile linear theory solution detailed earlier.

Whereas Euler pitch and yaw angles are undefined for point mass models, a good approximation is to assume that angle of attack is zero and the longitudinal axis of the projectile is tangent to the path.

\section{Low Launch Angle Trajectory}

The projectile used in this simulation is a representative direct fire fin-stabilized projectile $25 \mathrm{~mm}$ in diameter with six fins. The 
projectile weight, mass center measured along the stationline, roll inertia, and pitch inertia are $1.84 \mathrm{~N}, 0.118 \mathrm{~m}, 1.15 \times 10^{-5} \mathrm{~kg} \cdot \mathrm{m}^{2}$, and $2.78 \times 10^{-4} \mathrm{~kg} \cdot \mathrm{m}^{2}$, respectively. The projectile initial conditions are as follows: $x=0.0 \mathrm{~m}, y=0.0 \mathrm{~m}, z=0.0 \mathrm{~m}, \phi=0.0 \mathrm{deg}$, $\theta=2.0 \mathrm{deg}, \psi=0.0 \mathrm{deg}, \tilde{u}=762 \mathrm{~m} / \mathrm{s}, \tilde{v}=0.0 \mathrm{~m} / \mathrm{s}, \tilde{w}=0.0 \mathrm{~m} / \mathrm{s}$, $\tilde{p}=10.0 \mathrm{rad} / \mathrm{s}, \tilde{q}=0.0 \mathrm{rad} / \mathrm{s}$, and $\tilde{r}=0.0 \mathrm{rad} / \mathrm{s}$. The projectile is traveling through standard atmosphere without atmospheric wind.

Figures 3-6 show comparisons of the trajectories generated by the five prediction methods discussed. The standard linear theory and modified linear theory trajectories were computed using a dimensionless arc length step size of 100 calibers, which corresponds to a step of $2.5 \mathrm{~m}$ for this round and an initial time step of $0.003 \mathrm{~s}$ at the launch velocity. The six-degree-of-freedom and three-degreeof-freedom solutions were computed by using a time step size of $1.0 \times 10^{-5} \mathrm{~s}$.

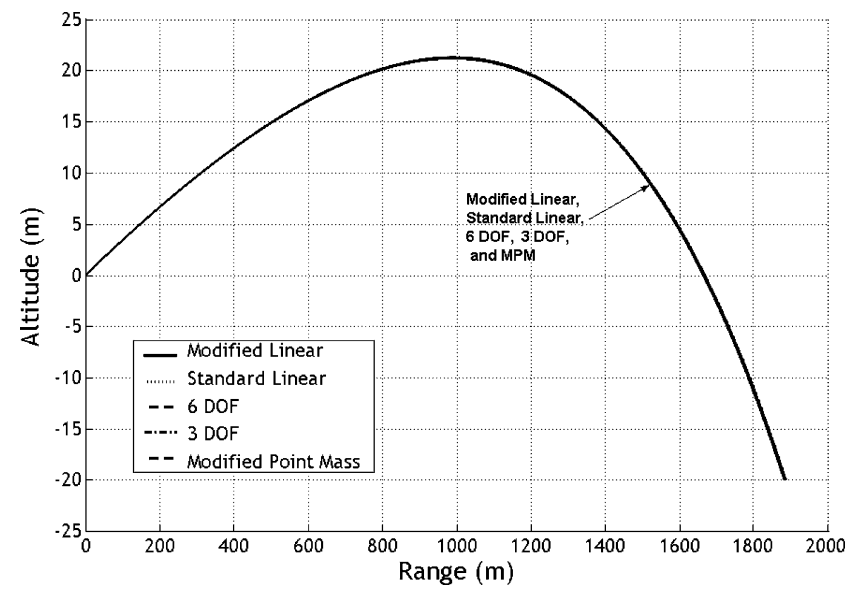

Fig. 3 Altitude vs range for low launch angle trajectory.

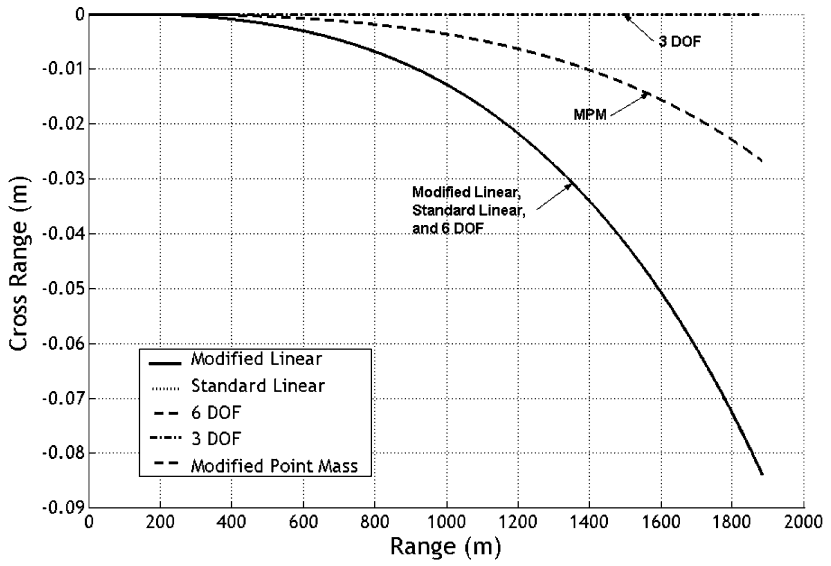

Fig. 4 Cross range vs range for low launch angle trajectory.

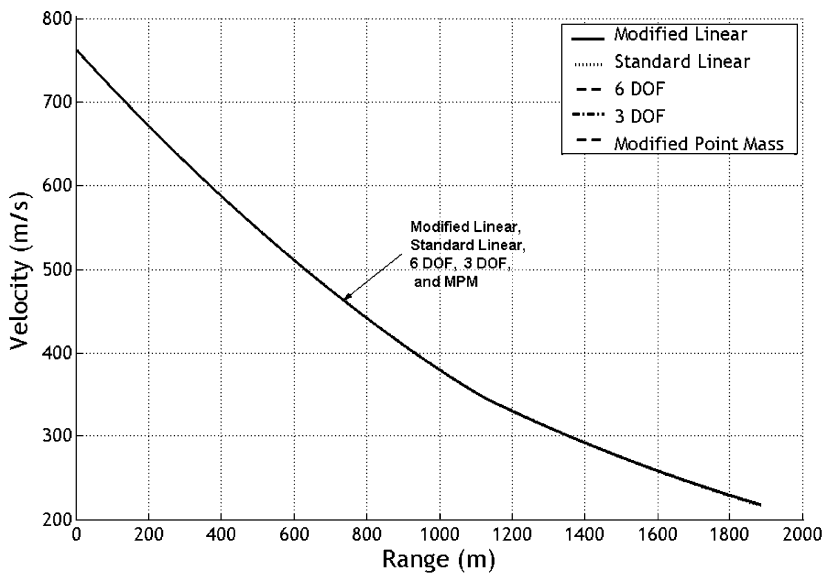

Fig. 5 Velocity vs range for low launch angle trajectory.

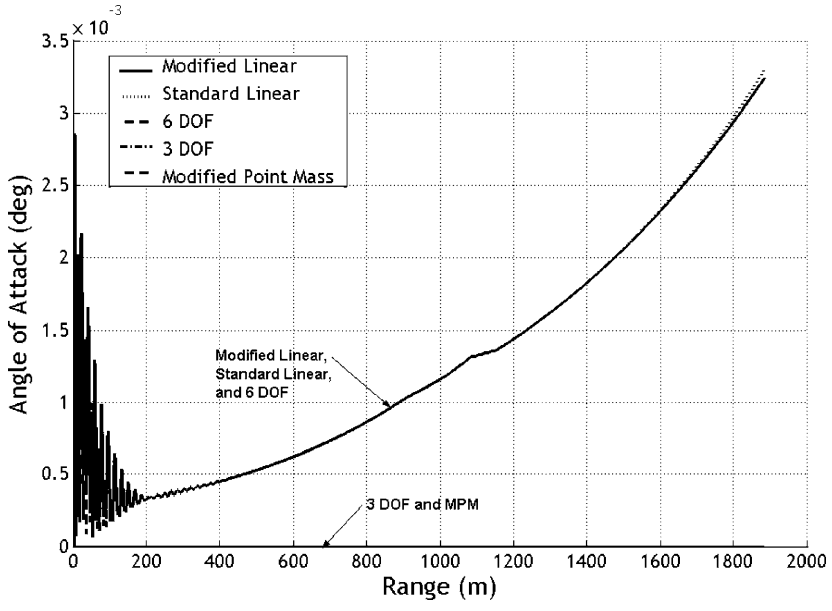

Fig. 6 Angle of attack vs range for low launch angle trajectory.

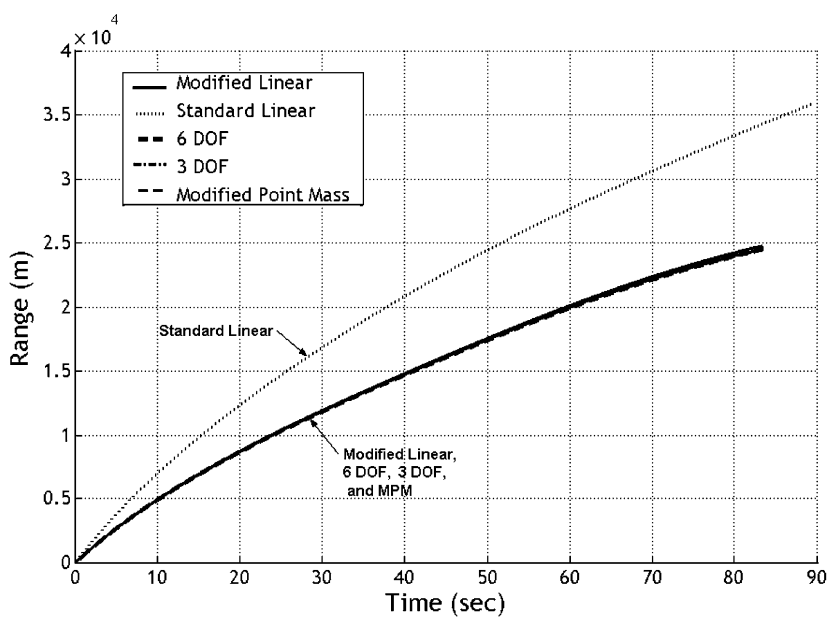

Fig. 7 Range vs time for high launch angle trajectory.

Figure 3 shows altitude vs downrange distance for each method. Both linear theory models and the three-degree-of-freedom model track the trajectory of the projectile predicted by the six-degree-offreedom model quite well. Figure 4 shows cross range vs downrange distance for each method. The linear theory models track projectile swerve predicted by the six-degree-of-freedom model, whereas the three-degree-of-freedom model predicts no swerve due to the exclusion of yaw of repose. Figure 5 shows velocity vs downrange distance, showing the linear theory models and the three-degreeof-freedom model match velocity predicted by the six-degree-offreedom model. Figure 6 shows angle of attack vs downrange distance. The modified and the standard linear theory models predict angle of attack well, whereas the three-degree-of-freedom model provides no angle-of-attack computation.

\section{High Launch Angle Trajectory}

The projectile used in this simulation is a representative indirect fire spin-stabilized projectile with a diameter of $155 \mathrm{~mm}$. The projectile weight, mass center measured along the stationline, roll inertia, and pitch inertia are $422 \mathrm{~N}, 0.324 \mathrm{~m}, 0.147 \mathrm{~kg} \cdot \mathrm{m}^{2}$, and $1.893 \mathrm{~kg} \cdot \mathrm{m}^{2}$, respectively. The projectile initial conditions are as follows: $x=0.0 \mathrm{~m}, y=0.0 \mathrm{~m}, z=0.0 \mathrm{~m}, \phi=0.0 \mathrm{deg}$, $\theta=45.0 \mathrm{deg}, \psi=0.0 \mathrm{deg}, \tilde{u}=853.4 \mathrm{~m} / \mathrm{s}, \tilde{v}=0.0 \mathrm{~m} / \mathrm{s}, \tilde{w}=0.0 \mathrm{~m} / \mathrm{s}$, $\tilde{p}=1800.0 \mathrm{rad} / \mathrm{s}, \tilde{q}=0.0 \mathrm{rad} / \mathrm{s}$, and $\tilde{r}=0.0 \mathrm{rad} / \mathrm{s}$. The projectile is traveling through standard atmosphere with a $6.1-\mathrm{m} / \mathrm{s}$ mean horizontal wind blowing from an angle of $57.3 \mathrm{deg}$ from the down range direction.

Figures 7-14 show a comparison of trajectories obtained by the five prediction methods discussed. Standard linear theory and modified linear theory trajectories were computed using a dimensionless arc length step size of 100 calibers, which corresponds to a step of 


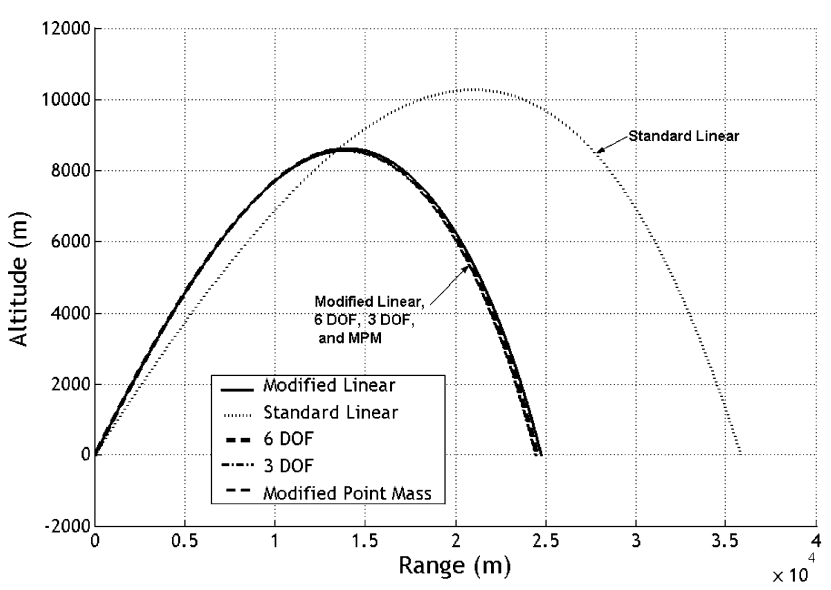

Fig. 8 Altitude vs range for high launch angle trajectory.

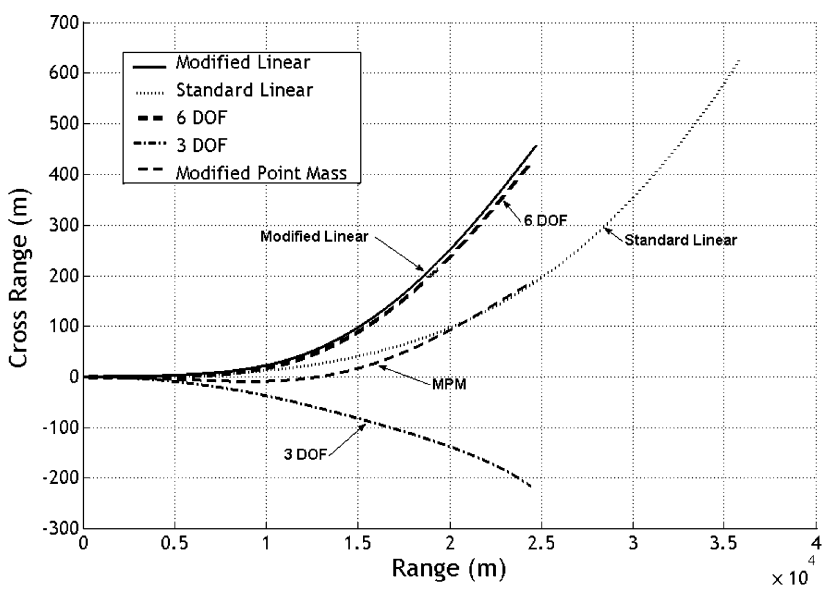

Fig. 9 Cross range vs range for high launch angle trajectory.

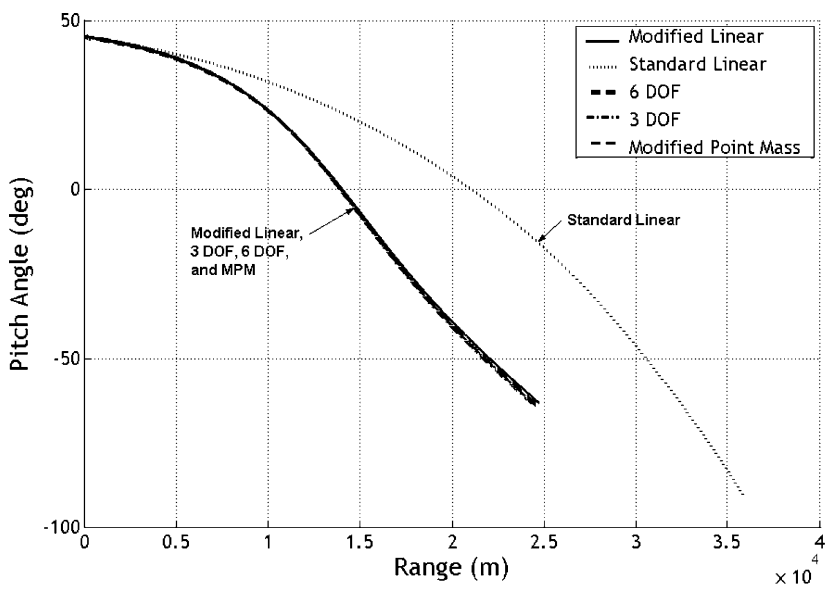

Fig. 10 Pitch angle vs range for high launch angle trajectory.

$15.5 \mathrm{~m}$ for this round and an initial time step of $0.018 \mathrm{~s}$ at launch velocity. The six-degree-of-freedom and three-degree-of-freedom numerical trajectories were computed by using a time step size of $1.0 \times 10^{-5} \mathrm{~s}$

For the high-angle shot, modified linear theory is able to predict the trajectory with reasonable accuracy, as shown in Figs. 7-14. Figures 7-9 show modified linear theory is able to predict the range, altitude, and projectile swerve in wind. Figures 10, 11, and 14 show it accurately accounts for high pitch angles, picks up yaw angle excursions, and predicts angle of attack. Figures 12 and 13 show that modified linear theory accurately predicts velocity and roll rate. Standard linear theory, limited by a low launch angle assumption, is unable to predict accurately the simulated trajectory, as seen in Figs. 7-14. One of the contributing factors for the inaccuracy of

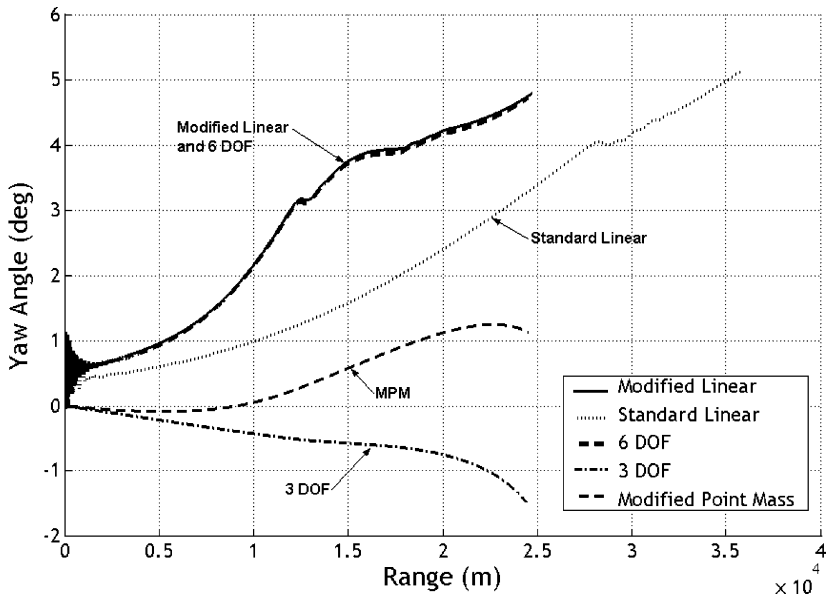

Fig. 11 Yaw angle vs range for high launch angle trajectory.

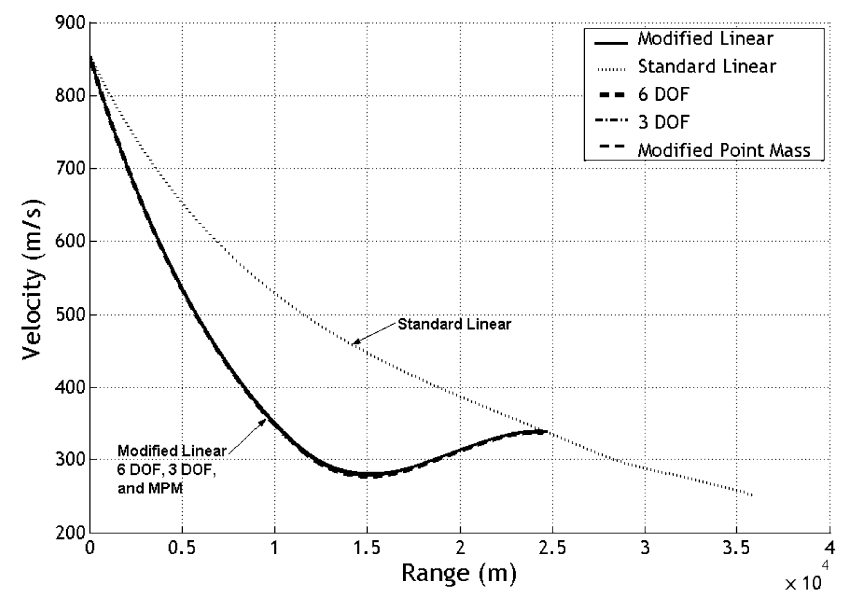

Fig. 12 Velocity vs range for high launch angle trajectory.

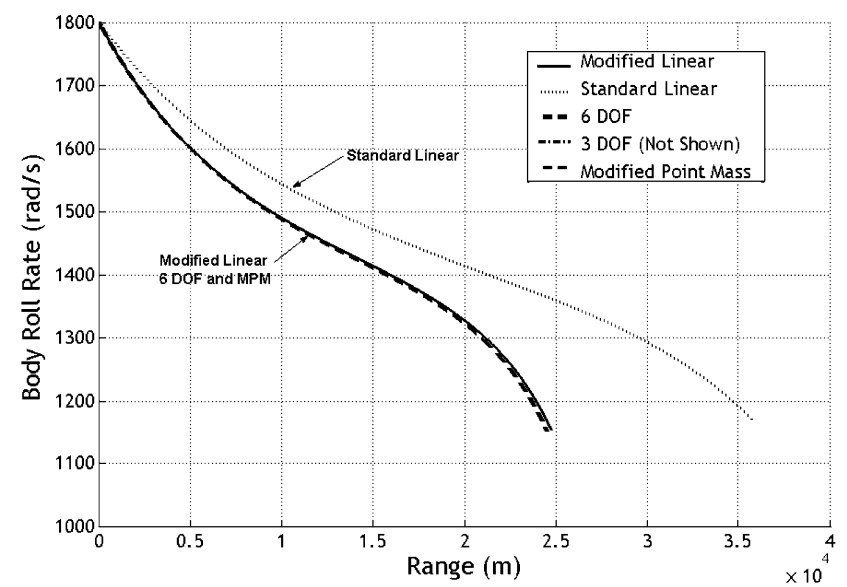

Fig. 13 Roll rate vs range for high launch angle trajectory.

standard projectile linear theory is the assumption of small Euler pitch angle. When pitch angle is not small, gravity tends to reduce total velocity of the round during ascent and increase total velocity during descent. The point mass models do not include rotational dynamics, and so any aerodynamic jump due to winds, initial pitch rate, initial yaw rate, etc., is not included in the trajectory results. In the high launch angle case, the atmospheric winds disturbance induces translational and angular rates to the projectile that in total tend to yaw the projectile to the right. The point mass models only include the effect of translational rates that tend to push the projectile left. In this case, projectile swerve is sensitive to angular rates leading to relatively large trajectory errors. The three-degree-of-freedom model is able to predict the range, altitude, and velocity reasonably 


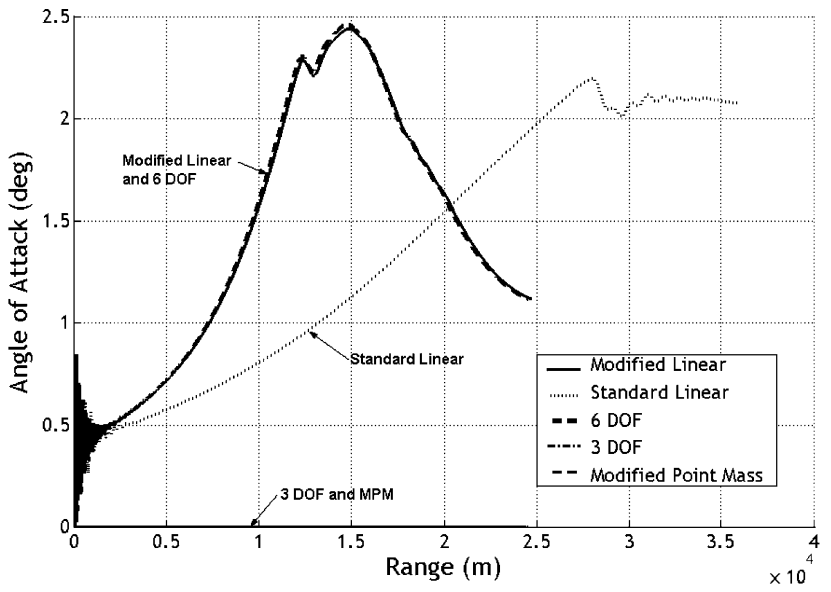

Fig. 14 Angle of attack vs range for high launch angle trajectory.

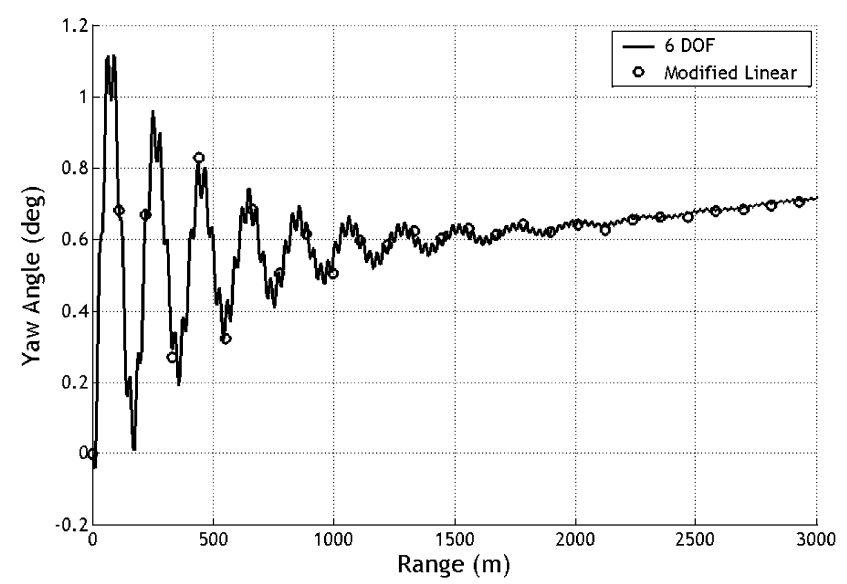

Fig. 15 Yaw angle vs range for a high launch angle with a large step size.

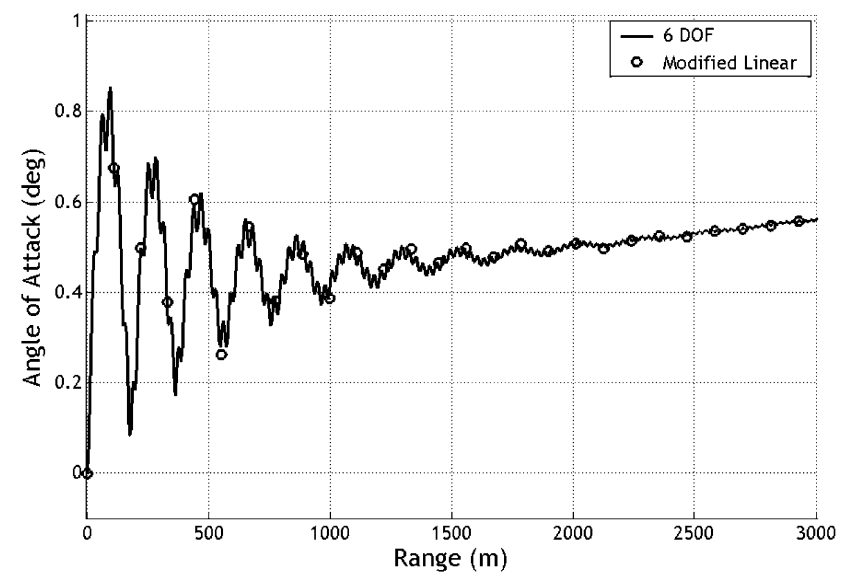

Fig. 16 Angle of attack vs range for a high launch angle with a large step size.

well, as seen in Figs. 7, 8, and 12, but is unable to generate proper projectile swerve as seen in Fig. 9, provides no information about the projectile's orientation as seen in Figs. 10 and 11, and angular rates, or angle of attack as shown in Fig. 14.

To highlight modified linear theory's ability to compute a trajectory rapidly, the high launch angle trajectory was recomputed using modified linear theory with a step size of 1000 calibers, or $155 \mathrm{~m}$. Figures 15 and 16 show a comparison of the yaw angle and the angle of attack predicted by the large step size modified linear theory case to those predicted by the nonlinear six-degree-of-freedom rigid-projectile model for the first $3000 \mathrm{~m}$ of the trajectory. The modified linear theory data in Figs. 15 and 16 is plotted as the discrete points calculated by each step. Figures 15 and 16 show that modified projectile linear theory can predict the trajectory using a step size that is large enough to skip over yaw cycles present in the projectile's motion. By not being limited in step size by system oscillations, modified projectile linear theory is able to compute rapidly future states, such as the impact point, using a small number of steps. This is due to the trajectory solution being generated from an approximate analytic closed-form solution and not numerical integration. Modified linear theory, although not quite as accurate as numerical integration of the nonlinear six-degree-of-freedom rigidprojectile model at small step sizes, is able to compute fairly accurate trajectories at step sizes much larger than those at which numeric integration becomes unstable. The modified projectile linear theory solution does not become unstable as the step size it increases. Rather, error in impact point prediction steadily increases.

To verify the computational efficiency of modified linear theory, a series of high launch angle trajectories were computed by modified projectile linear theory and a fourth-order Runge-Kutta numerical simulation of the six-degree-of-freedom model at different step sizes and the run times were recorded. A comparison of the times showed that for equivalent step sizes, the modified linear theory model computed the trajectory nearly twice as fast as the six-degree-of-freedom model.

\section{Conclusions}

This paper establishes a method for rapid and accurate computation of the trajectory of a projectile. Several other accurate methods exist for rapidly constructing projectile trajectories when the weapon is fired at low gun elevation angle. However, all existing methods exhibit relatively large trajectory errors for high gun elevation shots typical for indirect fire weapons. The modified projectile linear theory trajectory generator reported here should prove useful to smart weapon flight control system designers requiring real time in flight impact point estimates to determine control action.

\section{Acknowledgments}

The authors acknowledge the anonymous reviewers for their helpful comments on the initial manuscript.

\section{References}

${ }^{1}$ McCoy, R., Modern Exterior Ballistics, Schiffer, Attlen, PA, 1999, pp. 165-183, 240-244.

${ }^{2}$ Fowler, R., Gallop, E., Lock, C., and Richmond, H., "The Aerodynamics of a Spinning Shell," Philosophical Transactions of the Royal Society of London, Series A: Mathematical and Physical Sciences, Vol. 221, 1920.

${ }^{3}$ Kent, R., "An Elementary Treatment of the Motion of a Spinning Projectile About its Center of Gravity," U.S. Army Ballistic Research Lab., Rept. 85, Aberdeen Proving Ground, MD, 1937.

${ }^{4}$ Nielson, K., and Synge, J., "On the Motion of a Spinning Shell," U.S. Army Ballistic Research Lab., Rept. 116, Aberdeen Proving Ground, MD, 1943.

${ }^{5}$ Kelley, J., and McShane, E., "On the Motion of a Projectile with Small or Slowly Changing Yaw,” U.S. Army Ballistic Research Lab., Rept. 446, Aberdeen Proving Ground, MD, 1944.

${ }^{6}$ Kelley, J., McShane, E., and Reno, F., Exterior Ballistics, Univ. of Denver Press, Denver, CO, 1953.

${ }^{7}$ Hodapp, A., "Effect of Mass Asymmetry on Ballistic Match of Projectiles," Journal of Spacecraft and Rockets, Vol. 13, No. 12, 1976, pp. 757-760.

${ }^{8}$ Weber, D., "Simplified Method for Evaluating the Flight Stability of Liquid-Filled Projectiles," Journal of Spacecraft and Rockets, Vol. 31, No. 1, 1994, pp. 130-134.

${ }^{9}$ Murphy, C., "Angular Motion of a Spinning Projectile with a Viscous Liquid Payload," Journal of Guidance, Control, and Dynamics, Vol. 6, No. 4, 1983, pp. 280-286.

${ }^{10}$ Cobb, K. K., Whyte, R. H., and Laird, P. K., "The Effects of a Moving Components on the Motion of a 20-mm Projectile," 11th AIAA Aerodynamics Testing Conference, AIAA, New York, 1980, pp. 94-103.

${ }^{11}$ Hodapp, A., "Passive Means for Stabilizing Projectiles with Partially Restrained Internal Members," Journal of Guidance, Control, and Dynamics, Vol. 12, No. 2, 1989, pp. 135-139.

${ }^{12}$ Soper, W., "Projectile Instability Produced by Internal Friction," AIAA Journal, Vol. 16, No. 1, 1978, pp. 8-11. 
${ }^{13}$ Murphy, C., "Instability of Controlled Projectiles in Ascending or Descending Flight," Journal of Guidance and Control, Vol. 4, No. 1, 1981, pp. $66-69$.

${ }^{14}$ Cooper, G., "Influence of Yaw Cards on the Yaw Growth of Spin Stabilized Projectiles," Journal of Aircraft, Vol. 38, No. 2, 2001, pp. 266-270.

${ }^{15}$ Guidos, B., and Cooper, G., "Closed Form Solution of Finned Projectile Motion Subjected to a Simple In-Flight Lateral Impulse," AIAA Paper 20000767, 2000.

${ }^{16}$ Costello, M., and Peterson, A., "Linear Theory of a Dual-Spin Projectile in Atmospheric Flight," Journal of Guidance, Control, and Dynamics,
Vol. 23, No. 5, 2000, pp. 789-797.

${ }^{17}$ Burchett, B., Peterson, A., and Costello, M., "Prediction of Swerving Motion of a Dual-Spin Projectile with Lateral Pulse Jets in Atmospheric Flight," Mathematical and Computer Modeling, Vol. 35, No. 1-2, 2002, pp. $1-14$.

${ }^{18}$ Burchett, B., and Costello, M., "Model Predictive Lateral Pulse Jet Control of an Atmospheric Rocket," Journal of Guidance, Control, and Dynamics, Vol. 25, No. 5, 2002, pp. 860-867.

${ }^{19}$ Costello, M., and Anderson, D., "Effect of Internal Mass Unbalance on the Terminal Accuracy and Stability of a Projectile," AIAA Paper 96-3447, 1996. 